\title{
Adaptation de la microtechnique de fixation du complément au diagnostic de la peste équine
}

\author{
par G. BERNARD $\left(^{*}\right)$
}

RESUME

Adaptation de la microtechnique de fixation du complément au diagnostic de la peste équine

\begin{abstract}
Afin de déterminer rapidement l'immunité d'une population équine, il étajt nécessaire de posséder une méthode simple. L'auteur a fait une adaptation à la peste équine de la microtechnique de fixation du complément, en précisant les différents paramètres. Cette épreuve doit être utilisée pour une recherche statistique uniquement et pour des cas d'immunisation récente.
\end{abstract}

\section{INTRODUCTION}

La peste équine sévit toujours à l'état endémique en Afrique. Il est même vraisemblable qu'elle diffuse vers le Nord à partir de foyers silencieux centre-africains mis en évidence par MAURICE et PROVOST (6). D'autre part, BOURDIN, BERNARD et LAURENT (1974) ont montré l'existence d'une forte proportion d'anticorps parmi les équins du Sénégal. Les échanges entre l'Afrique et l'Europe s'accentuant, un marché des chevaux africains est en train de se développer.

Aussi, il a paru nécessaire de mettre au point une méthode rapide et commode de recherche des anticorps dans le serum.

Il existe 8 types principaux d'antigène Peste equine, il fallait donc s'adresser à une épreuve de groupe telle la fixation du complément (5).

La microméthode a été retenue car elle présente une commodité d'emploi appréciable et permet de faire un grand nombre d'examens simultanément.

(*) Laboratoire National de l'Elevage et de Recherches Vétérinaires, B.P. 2057, Dakar-Hann. République du Sénégal.

\section{MATERIEL ET METHODE}

\section{A) Les milieux}

Les suspensions virulentes utilisées pour les inoculations intracérébrales de souris sont réalisées dans un tampon phosphaté pH 7,4 dont la formule est la suivante (1) :

- phosphate disodique $\left(\mathrm{Na}_{2} \mathrm{HPO}_{4}\right)$ anhydre . . $3,20 \mathrm{~g}$

- Phosphate monosodique $\mathrm{NaH}_{2} \mathrm{PO}_{4}, 2 \mathrm{H}_{2} \mathrm{O}$ ) cristallisé $0,33 \mathrm{~g}$

- Chlorure de sodium. . . $6 \mathrm{~g}$

- Eau bidistillée Q.S.P. . . $1000 \mathrm{ml}$

Stériliser à l'autoclave à $120^{\circ} \mathrm{C}$ pendant 20 minutes.

Les hématies sont récoltées dans du citrate de soude ou dans une solution de Alsever.

Les sérums, antigènes et complément sont dilués dans une solution de Veronal Gelatine (VG) :

- 10 comprimés « Complement fixation test diluent tablets » (oxoïd) .

- Gelatine (Difco) . . . . . $1 \mathrm{~g}$

- Eau bidistillée Q.S.P. . . . $1000 \mathrm{ml}$ 
Dissoudre au bain-marie à chaud.

Lorsqu'on prépare une gamme d'hémolyse, on utilise une solution de Veronal 5 fois concentrée (V5) préparée en dissolvant à chaud un comprimé «Oxoî̀ » dans $20 \mathrm{ml}$ d'eau bidistillée.

Tous ces milieux sont conservés à $+4^{\circ} \mathrm{C}$.

\section{B) Le matériel spécial}

A côté du matériel habituel, on utilise des microdilueurs de $0,025 \mathrm{ml}$, des pipettes compte-gouttes des $0,025 \mathrm{ml}$, des microplaques en $\mathrm{U}$, des porte-plaques pour centrifugation et du papier GO.NO.GO pour contrôle des microdilueurs.

Ces instruments font partie du Microtiterkit produit par Cooke Instruments.

\section{C) Préparation des différents constituants :}

1. L'antigène: la fixation du complément peste équine étant une épreuve de groupe, on pourra utiliser l'un quelconque des types. Néanmoins, le type 9-S2 convient particulièrement.

L'antigène est préparé sur cerveaux de souris âgées de trois semaines environ.

On inocule, à chaque animal, $0,03 \mathrm{ml}$ par voie intracérébrale, d'une dilution au $1 / 20$ dans du tampon phosphaté pH 7,4 de la souche neurotrope conservée lyophilisée à $-25^{\circ} \mathrm{C}$. Au bout de 3 à 5 jours, les souris présentant des symptômes nerveux très importants et une paralysie presque complète sont anesthésiées. Après découpage de la boîte crânienne, les cerveaux seront técoltés et congelés. Après décongélation, ils sont lavés trois fois dans du VG. On prépare une suspension au 1/20 (10 cerveaux $+12 \mathrm{ml}$ de VG) que l'on broie dans un mixer. La suspension est centrifugée $1 \mathrm{~h}$ à $10000 \mathrm{t} / \mathrm{mn}$ à $+4^{\circ} \mathrm{C}$. Le surnageant représente l'antigène. Il peut être utilisé tel quel (le conserver à $+4^{\circ} \mathrm{C}$ ) ou bien inactivé par $0,1 \mathrm{ml}$ de solution de betapropiolactone à 0,1 p. 100 dans de l'eau distillée pour $0,9 \mathrm{ml}$ d'antigène (agitation de $3 \mathrm{~h}$ à température ambiante puis $18 \mathrm{~h}$ à $+4^{\circ} \mathrm{C}$ ).

Les antigènes préparés de cette manière ne présentent aucun pouvoir anticomplémentaire s'ils sont utilisés dans un délai de 1 mois.

2. Les hématies : on utilise des hématies de mouton. Le sang est prélevé dans une quantité égale de citrate de soude ou de Alsever. On le conserve 4 à 5 jours à $+4^{\circ} \mathrm{C}$ avant l'utilisation. Les hématies sont alors lavées trois fois après centrifugation, avec la solution VG. Le culot du dernier lavage est remis en suspension dans un volume égal de VG. Conservation d'une semaine à $+4^{\circ} \mathrm{C}$.

3. Le sérum hémolytique : on utilise le sérum de lapin anti-hématies de mouton.

Dans toutes les épreuves, on utilise une solution d"hémolysine (SH) diluée au 1/500 (2 microgouttes de $\mathrm{SH}$ dans $12 \mathrm{mI}$ de VG) réalisant ainsi les conditions d'excès d'hémolysine.

4. Préparation du stock d'hématies sensibilisées (SS-SH) : on prépare une suspension à 3 p. 100 d'hématies (SS) dans la solution de VG à laquelle on ajoute, en effectuant un mouvement rotatif, un volume identique de $\mathrm{SH}$ (au $1 / 500$ ). Après incubation de $15 \mathrm{mn}$ à température ambiante, la suspension de SS-SH peut être conservée $24 \mathrm{~h}$ à $+4^{\circ} \mathrm{C}$.

5. Préparation de la gamme d'hémolyse: suivant BARME et Collaborateurs (1) il est nécessaire de disposer d'une échelle étalon d'hémolyse avec laquelle des comparaisons pourront être faites. On prépare:

a) une solution d'hémoglobine (solution A) contenant $1 \mathrm{ml}$ de SS $+7 \mathrm{ml}$ d'eau bidistillée. Après agitation, on ajoute $2 \mathrm{ml}$ de solution de V5.

b) Une suspension d'hématies (suspension B) en ajoutant $9 \mathrm{ml}$ de VG à $1 \mathrm{ml}$ de SS. On répartit dans 11 tubes à hémolyse :

Solution A : 0,0 - 0,1 - 0,2 - 0,3 - 0,4 - 0,5 $0,6-0,7-0,8-0,9-1,00 \mathrm{ml}$

Solution B : $1,00-0,9-0,8-0,7-0,6-0,5-$ $0,4-0,3-0,2-0,1-0 \mathrm{ml}$

Pourcentage d'hémolyse : 0 - 10 - 20 - 30 - 40 $50-60-70-80-90-100$

Après agitation on reporte 5 gouttes à 0,025 $\mathrm{ml}$ de chaque pourcentage dans les cupules d'une micro-plaque. On centrifuge 5 à 1500 $\mathrm{t} / \mathrm{mn}$ à $+4^{\circ} \mathrm{C}$. On recouvre de cellophane adhésive et l'on peut conserver plusieurs jours à $+4^{\circ} \mathrm{C}$.

\section{D) Titrage des différents constituants}

1. Le complément : on utilise dans la réac- 


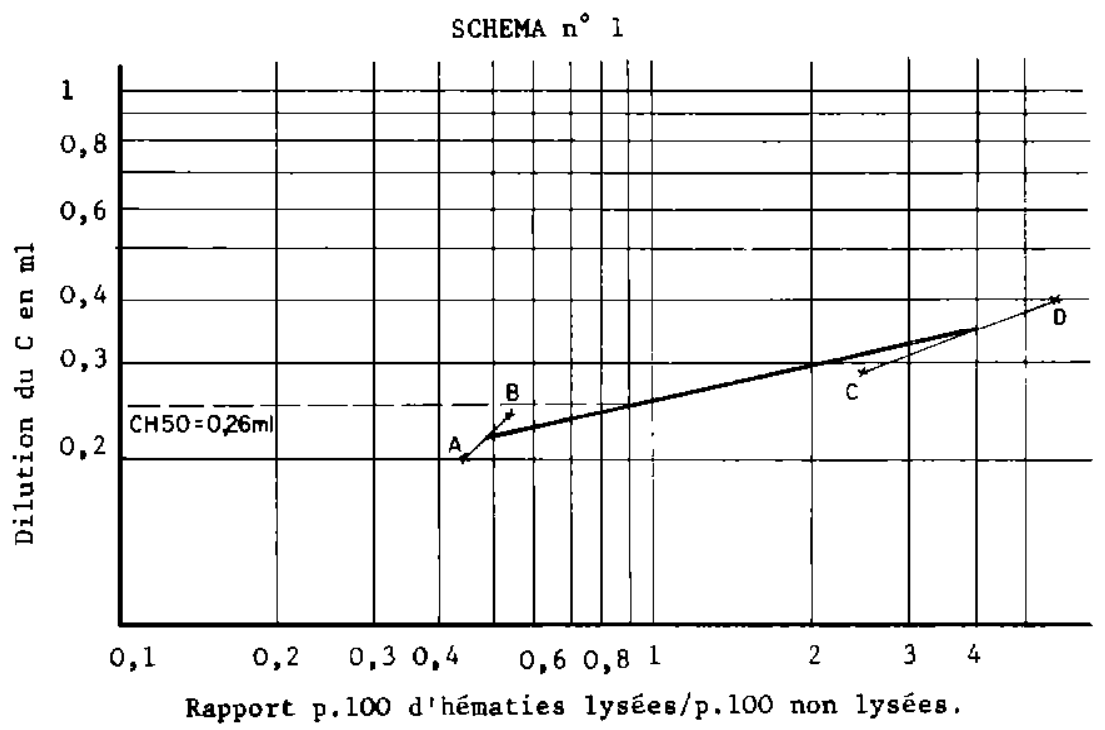

Schéma no 1. - Recherche de l'unité de complément hémolyse 50 p. 100.

tion 5 unités de complément hémolyse 50 p. 100. On prépare deux solutions mères dans du VG : l'une au 1/250 (solution A) et l'autre au $1 / 300$ (solution $B$ ). Si le complément est très actif, préparer les solutions mères au $1 / 400$ et $1 / 450$. On fait les dilutions suivantes en tubes à hémolyse :

$\begin{array}{cccccc}\text { Tube } & & \mathrm{N}^{*} 1 & 2 & 3 & 4 \\ \text { VG } & & 0,60 & 0,55 & 0,50 & 0,40 \\ \text { ution } & \text { A } & 0,20 & 0,25 & 0,30 & 0,40 \\ \text { Tube } & & \text { N }^{\circ} 5 & 6 & 7 & 8 \\ \text { VG } & & 0,60 & 0,55 & 0,50 & 0,40 \\ \text { ution } & \text { B } & 0,20 & 0,25 & 0,30 & 0,40\end{array}$

On effectue le titrage en quadruple sur une plaque. On reporte 4 gouttes de $0,025 \mathrm{ml}$ de chacun des tubes 1 à 8 et on ajoute $0,025 \mathrm{ml}$ de SS-SH dans chaque cupule. Après agitation, on met $30 \mathrm{mn}$ à l'étuve à $37^{\circ} \mathrm{C}$ avec agitation à la $15^{\mathrm{e}}$ minute. On centrifuge alors $5 \mathrm{mn}$ à $1500 \mathrm{t} / \mathrm{mn}$ à $+4^{\circ} \mathrm{C}$.

Les quatre titrages doivent donner des résultats identiques, sinon l'on prend la moyenne. On établit les courbes d'hémolyse sur papier logarithmique pour $1 / 250$ et $1 / 300$ (schéma $\mathrm{n}^{\circ} 1$ ). Les points $\mathrm{A}, \mathrm{B}, \mathrm{C}, \mathrm{D}$ de la courbe auront pour ordonnée le volume de complément dans les tubes 1 à 4 (pour $1 / 250$ et 5 à 8 (pour $1 / 300$ ). Les abscisses seront données par les valeurs suivantes correspondant aux pourcentages d'hémolyse :

Pourcentage d'hémolyse : $10-20-30-40$ $50-60-70-80-90$.
Abscisses : 0,111 - 0,25 - 0,43 - 0,67 - 1,00 $1,50-2,33-4,00-9,00$.

suivant les valeurs données par WORK et HAMMON (9). Puis on joint d'une part AB et d'autre part $C D$. On trace la droite passant par le milieu de $\mathrm{AB}$ et de $\mathrm{CD}$. Elle coupe la ligne logarithmique 1,00 en un point dont l'ordonnée $\mathrm{Y}$ sert au calcul suivant :

$$
\frac{5 \mathrm{Y}}{250 \text { (ou 300) }}=\frac{0,4}{\mathrm{X}}
$$

$0,05 \mathrm{ml}$ d'une dilution à $1 / \mathrm{X}$ contiendront les 5 unités 50 p. 100.

2. L'antigène : On recherche l'optimum antigénique, on prépare, d'une part 6 tubes à hémolyse contenant les dilutions d'un sérum positif connu décomplémenté à $60^{\circ} \mathrm{C}$ pendant $30 \mathrm{mn}$ (dilutions du $1 / 4$ au $1 / 128$ ) et d'autre part 6 tubes contenant les dilutions de l'antigène (du $1 / 2$ au 1/64). On utilise une microplaque (schéma $\mathbf{n}^{\circ}$ 2) dans laquelle on distribue dans 6 rangées verticales les dilutions du sérum, à raison de $0,025 \mathrm{ml}$ par cupule et dans 7 cupules par rangée (la dernière qui est le témoin sérum sert à contrôler un éventuel pouvoir anticomplémentaire dans chaque dilution).

Dans 6 rangées horizontales, on distribue les dilutions de l'antigène à raison de 7 cupules par dilution et $0,025 \mathrm{ml}$ par cupule (la dernière étant le témoin antigène). Dans les 2 rangées témoins antigène et sérum, on ajoute $0,025 \mathrm{ml}$ de solution de VG par cupule. On distribue $0,05 \mathrm{ml}$ de VG dans 3 cupules qui serviront de contrôle complément et $0,1 \mathrm{ml}$ dans une cupule qui sera le témoin hématies. 


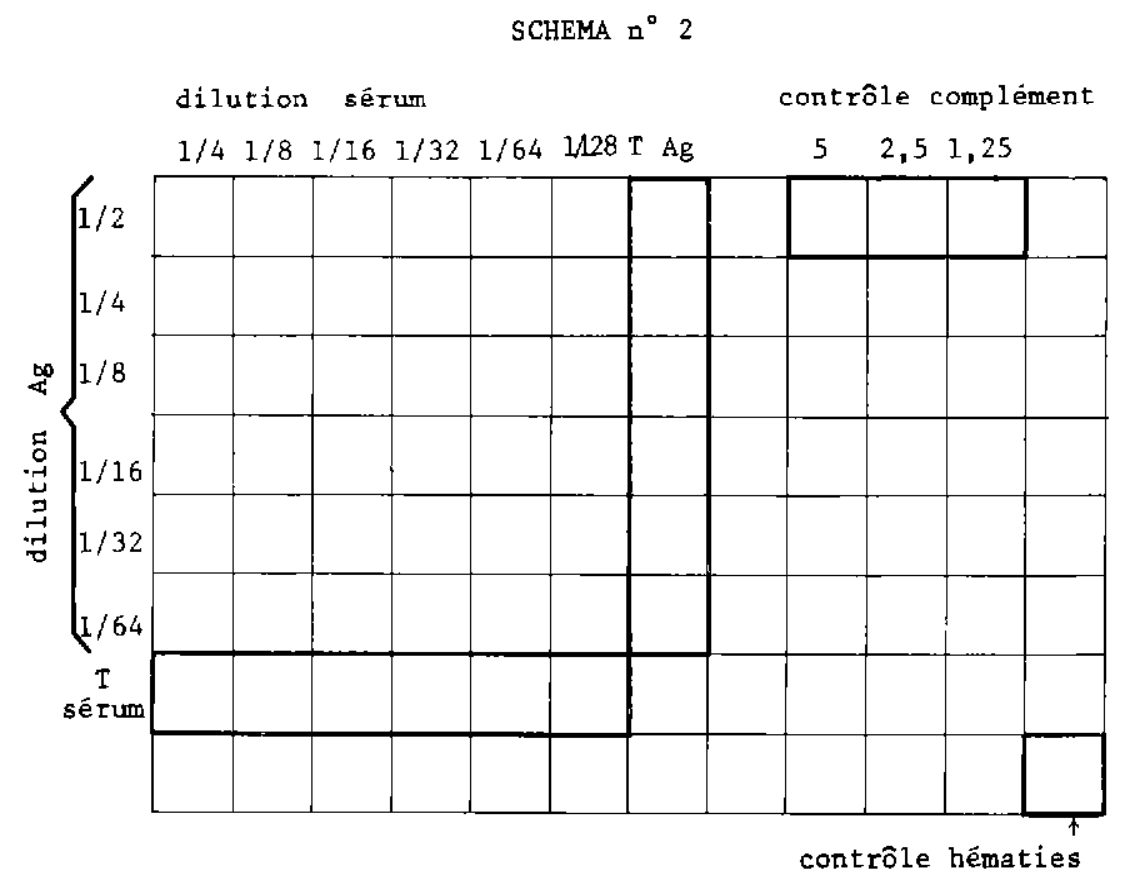

Schéma $n^{\circ}$ 2. - Recherche de l'optimum antigénique: disposition des différents constituants sur la microplaque.

On agite et on laisse $15 \mathrm{mn}$ à température ambiante, temps pendant lequel on prépare les dilutions du complément:

a) à 5 unités - $6 \mathrm{ml}$ selon la dilution déterminée par le titrage.

b) à 2,5 unités $-1 \mathrm{ml}$ à 5 unités $+1 \mathrm{ml}$ de VG.

c) à 1,25 unités - $0,5 \mathrm{ml}$ à 5 unités + $1,5 \mathrm{ml}$ de VG.

Le complément est alors distribué dans toutes les cupules $(0,05 \mathrm{ml}$ par cupule) sauf dans le témoin hématies:

- à 5 unités dans toutes les cupules réaction.

- à 5 , à 2,5 et à 1,25 unités dans les trois cupules témoins complément.

Après agitation, on place à $+4^{\circ} \mathrm{C}$ pendant 18 heures.

La plaque est ensuite remise à température ambiante pendant $15 \mathrm{mn}$ puis on distribue dans toutes les cupules $0,025 \mathrm{ml}$ de SS-SH. Après $30 \mathrm{mn}$ à $37^{\circ} \mathrm{C}$ (agitation à $15 \mathrm{mn}$ ) on centrifuge $5 \mathrm{mn}$ à $1500 \mathrm{t} / \mathrm{mn}$ à $+4^{\circ} \mathrm{C}$. On doit observer les pourcentages suivants d'hémolyse dans les témoins :

— contrôle complément 5 unités : 100 p. 100

- contrôle complément 2,5 unités : 90 100 p. 100
- contrôle complément 1,25 unités : 40 75 p. 100

— témoins Antigène : 100 p. 100

— témoins Sérums : 100 p. 100

— témoins Hématie : 0 p. 100

Le titre sera donné par la dilution de l'antigène ayant fourni le plus haut titre au sérum (courbe à 30 p. 100 d'hémolyse).

\section{E. Exécution de la réaction de fixation du complément (photo $n^{0}$ 1)}

Les sérums à examiner sont dilués au 1/4 dans la solution de VG et décomplémentés à $60^{\circ} \mathrm{C}$ pendant $30 \mathrm{mn}$. Pendant ce temps les micro-dilueurs sont contrôlés sur papier GO. NO.GO.

On distribue pour chaque sérum $0,025 \mathrm{ml}$ de VG dans 4 cupules d'une rangée verticale (les trois premières contiendront des dilutions du sérum allant du $1 / 8$ au $1 / 32$ et la $4^{e}$ servira de témoin sérum au $1 / 8$ afin de contrôler un éventuel pouvoir anticomplémentaire).

Les dilutions se font pour quatre sérums simultanément, à l'aide des microdilueurs que l'on reporte après les avoir trempés dans les solutions au 1/4 dans les premières cupules de 4 rangées verticales. Après rotation, on passe 


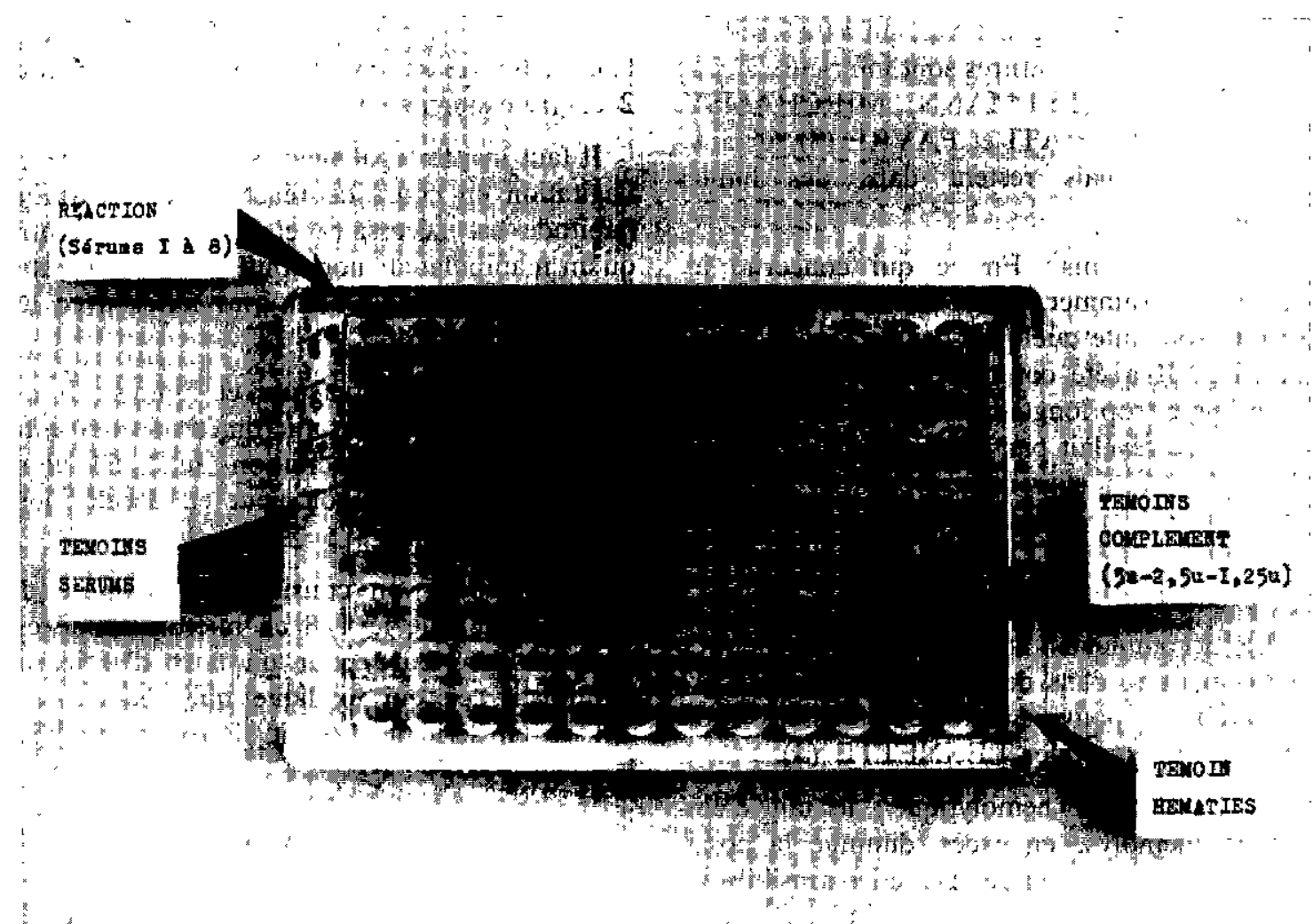

Photo no 1. - Epreuve de fixation du complément sur microplaque.

- sérum sans anticorps: $n^{\circ} 6$

- sérum ayant des anticorps au $\frac{1}{8}: n^{\prime \prime} 2$
- sérum ayant des anticorps au $\frac{1}{16}: n^{\text {us } 1-3-4}$

- sérum ayant des anticorps au $\frac{1}{32}: n^{0}$ 5-7-8 aux deuxièmes cupules puis aux troisièmes. On retrempe alors les dilueurs dans les dilutions initiales au $1 / 4$ et on les porte dans les cupules témoins. Puis on distribue dans les cupules témoins, $0,025 \mathrm{ml}$ de VG. $0,05 \mathrm{ml}$ sont distribués dans trois cupules témoins complément (5 - 2,5 1,25 unités) et $0,1 \mathrm{ml}$ dans un témoin hématie.

L'antigène titré est dilué et distribué dans les 3 premières cupules des rangées verticales $(0,025 \mathrm{ml}$ par cupule). Après agitation on laisse $15 \mathrm{mn}$ à température ambiante.

Le complément dilué est ensuite distribué ( $0,05 \mathrm{ml}$ par cupule) :

- à 5 unités dans toutes les cupules réactions, à $5,2,5$ et 1,25 unités dans les témoins correspondants.

Après agitation, on met à $+4^{\circ} \mathrm{C}$ pendant $18 \mathrm{~h}$. Ensuite, on stabilise $15 \mathrm{mn}$ à température ambiante, on distribue dans toutes les cupules $0,025 \mathrm{ml}$ de SS - SH.

Après agitation, les plaques sont mises $30 \mathrm{mn}$ à l'étuve à $37^{\circ} \mathrm{C}$ (agitation à la $15^{\mathrm{e}}$ minute), puis elles sont centrifugées $5 \mathrm{mn}$ à $1500 \mathrm{t} / \mathrm{mn}$ à $+4^{\circ} \mathrm{C}$.

Le titre des sérums est indiqué par la dilution la plus élevée donnant de 0 à 30 p. 100 d'hémolyse.

\section{RESULTATS ET DISCUSSION}

\section{A. Les différents constituants}

1. L'antigène: la souche neurotrope utilisée pour préparer l'antigène doit être diluée au $1 / 20$ minimum. Plusieurs essais faits avec des concentrations virales plus importantes se sont révélés négatifs, les souris inoculées résistant à l'injection. D'autre part, deux années d'expérimentation sur l'antigène de cerveau non préparé comme indiqué dans la technique classique de CLARKE et CASALS (3), ont montré que cet antigène utilisé dans le mois suivant sa préparation ne présentait pas de pouvoir anti- 
complémentaire et conservait un titre oscillant autour du $1 / 16$. Ces titres sont inférieurs à ceux obtenus par STELLMANN, MIRCHAMSY, GIRAUD, HAZRATI et FAVRE (8) sur antigène traité mais restent dans des limites acceptables.

2. Les sérums: En ce qui concerne les sérums à examiner, il est nécessaire que leur récolte soit faite quelques heures après la prise de sang. Il a été constaté que le sérum d'un sang laissé trop longtemps au repos avant l'extraction se révélait par la suite anticomplémentaire plus particulièrement en ce qui concerne les sérums d'âne ainsi que l'ont montré PILOMORON, VINCENT, AIT-MESBAH et FORTHOMME (7).

La décomplémentation à $60^{\circ} \mathrm{C}$ au lieu de $56^{\circ} \mathrm{C}$ permet, dans de nombreux cas, de faire disparaître le pouvoir anticomplémentaire comme le fait remarquer LENNETTE (4).

3. Le système hémolytique: le fait d'employer l'hémolyse en excès, diminue la possibilité de conservation des hématies sensibilisées qui s'agglutinent, aussi, vaut-il mieux utiliser une suspension fraîche de SS - SH pour chaque réaction.

\section{B. L'épreuve de fixation du complément et sa valeur}

1. Technique: la microtechnique utilisée s'est révélée très pratique. 200 sérums peuvent être analysés en une seule épreuve quantitative. L'ensemble des manipulations est réalisable en deux heures par un seul technicien. La lecture est assez aisée si la centrifugation est faite.

La reproductibilité statistique est également bonne mais il faut noter que d'une manière individuelle, ainsi que l'a montré BERNARD (2), des différences apparaissent d'une épreuve à l'autre. On ne saurait donc trop recommander 2 contre-examens en cas de doute.

Il faut insister également sur l'importance de l'agitation en cours de réaction. Une certaine habitude est nécessaire et il a été démontré qu'un manipulateur non confirmé pouvait rater successivement plusieurs séries faites avec les mêmes sérums.

2. Portée de la réaction et limites: Il est très rare de déceler en microméthode des anticorps au-delà d'une dilution au 1/64 alors qu'en macrométhode on peut en déceler jusqu'au $1 / 128$ (5).

D'autre part, on considère en matière de peste équine que le $1 / 8$ en microméthode est la limite inférieure pour un taux d'anticorps significatif, ce qui explique que seuls soient utilisés en série le $1 / 8$, le $1 / 16$ et le $1 / 32$.

\section{CONCLUSION}

La microméthode de fixation du complément peste équine est une épreuve de groupe pouvant être utilisée facilement par un laboratoire non spécialisé. Utilisant des antigènes inactivés, elle peut servir au contrôle des anticorps dans un pays réputé non infecté.

Son emploi requiert une manipulation méthodique par un agent entrainé.

C'est une réaction très intéressante lorsqu'on veut faire le survol de l'immunité récente d'une population équine, mais dans tous les autres cas il sera nécessaire de faire appel à d'autres techniques telle la séroneutralisation sur sourīs ou la recherche de l'index de neutralisation sur cultures cellulaires.

\section{SUMMARY}

Adaptation of the microtechnique complement fixation test for the diagnosis of african horse sickness

To determine the immunity of a large equine population, it was necessary to have a rapid as well as simple method. The author has done this adaptation of the microtechnique complement fixation test to african horse sickness by precising the different conditions and reagents used in the test.

This test should be applied only for the purpose of statistical research, and in case of recent immunization. 


\section{RESUMEN}

\section{Adaptación de la microtécnica de fijación del complemento para el diagnóstico de la peste équina.}

Para determinar rapidamente la inmunidad de una población de caballos, necesitaba tener un método sensillo. El autor ha probado la microtécnica de fijación del complemento para la peste equina, precisando las diferentes condiciones de uso. Esta técnica sirve unicamente para la búsqueda estadística y para casos de inmunización reciente.

\section{BIBLIOGRAPHE}

1. BARME (M.), BRES (P.), HERY (G.), ROBIN (Y). Techniques des laboratoires des virus et des arbovirus. Rapport fonct. Tech. Inst. Pasteur Da$k a r, 1969-1970$ : 159-244.

2. BERNARD (G.). Les anticorps neutralisant et fixant le complément dans la peste équine africaine. 3e Congrès de virologie. Madrid, 10-17 septembre 1975.

3. CLARKE (D. H.), CASALS (J.). Techniques for hemagglutination and hemagglutination inhibition with arthropod-borne viruses. Am. J. trop. Med. Hyg., 1958, $7: 561$.

4. LENNETTE (E. H.). General principles underlying laboratory diagnosis for viral and rickettsial infections. Diagnostic procedures for viral and rickettsial diseases, 1964 : ch. I.

5. Mc INTOSH (B. M.). Complement fixation with horse sickness. Onderstepoort J. vet. Res., 1956, 27: 165-169.

6. MAURICE (Y.), PROVOST (A.). La peste équine à type 9 en Afrique centrale. Enquête sérologique. Rev. Elev. Méd. vét. Pays trop., 1967, 20 (1) : 21-25.

7. PILO-MORON (E.), VINCENT (J.), AIT-MESBAH (O.) et FORTHOMME (G.). Origine de la peste équine en Afrique du Nord: Résultats d'une enquête sur les ânes du Sahara algérien. Arch. Inst. Pasteur, Algérie, 1969, 47: 105-118.

8. STELLMAN (C.), MIRCHAMSY (H.), GIRAUD (M.), HAZRATI (A.) et FAVRE (H.). Note sur le pouvoir fixant le complément du virus peste équine. Rec. Méd. vét., 1969, $145: 1267-1282$.

9. WORK (T. H.), HAMMON (W. Mc D.). Arbovirus infection in man. Diagnostic procedures for viral and rickettsial diseases, $1964: \mathrm{ch} .8$. 